\section{Commentary: Lung cancer and dysbiosis: Debugging the studies for the future}

\author{
Harvey I. Pass, MD
}

Thoracic oncologists have enough on their collective mind trying to remember genomic or epigenomic targets for lung cancer, the newest checkpoint molecule to neutralize, or some immunologic phenomenon of the tumor microenvironment that explains progressors from nonprogressors after resection. As detailed by Patnaik and colleagues, ${ }^{2}$ we must now recognize that the mucosal lined upper and lower airway has a microbiome that is not only involved with chronic obstructive pulmonary disease ${ }^{3}$ and tuberculosis, ${ }^{4,5}$ but also may play a crucial part as a promoter of lung cancer. ${ }^{1,6-9}$ These investigators used archived saliva, tumor, lung cancer tissue, and matching lung from different specimen banks to classify the bacteria involved in the lung cancer microbiome (LCM). Using state of the art multiomic next-generation sequencing platforms, they report a dysbiotic signature and associated pathways with lung cancer recurrence. Their studies add credibility to the hypothesis that microaspiration influences the LCM because bronchoalveolar lavages (BALs) with high amylase levels had bacteria very similar to those seen in saliva, validate previous studies regarding the abundance as well as the diversity of bacteria at different sites in the LCM, and present a novel 19-genera signature that predicted recurrence with an accuracy of $89 \%$ ! Phenomenal work. But don't think about novel trials with antibiotics yet. Here's why.

The study of the LCM is in its infancy, and the rules of the game have not been standardized. The study cohorts are as varied as the number of publications: some studies compare microbiome differences between lung cancer populations and healthy individuals using BALs, brushes, and saliva ${ }^{10}$ whereas others use paired involved/uninvolved BALs

From the Division of General Thoracic Surgery, Department of Cardiovascular Surgery, NYU Langone Medical Center, New York, NY.

Disclosure: Author has nothing to disclose with regard to commercial support.

Received for publication Jan 26, 2020; revisions received Jan 26, 2020; accepted for publication Feb 4, 2020; available ahead of print March 19, 2020.

Address for reprints: Harvey I. Pass, MD, Division of General Thoracic Surgery, Department of Cardiothoracic Surgery, NYU Langone Medical Center, 530 First

Ave, 9V, New York, NY 10016 (E-mail: Harvey.Pass@nyumc.org).

J Thorac Cardiovasc Surg 2021;161:430-1

$0022-5223 / \$ 36.00$

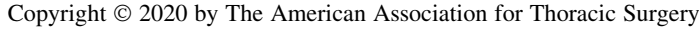

https://doi.org/10.1016/j.jtcvs.2020.02.127

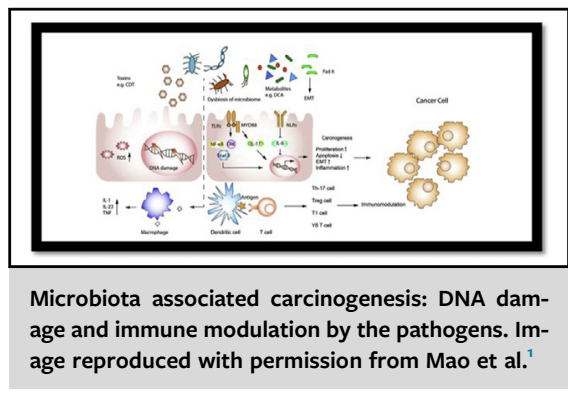

CENTRAL MESSAGE

The lung microbiome has enor-

mous ramifications for lung can-

cer if we can avoid specimen

contamination, harmonize

computational techniques, and

uncover dysbiotic mechanisms

for cancer promotion.

from patients with documented lung cancer. ${ }^{11}$ A minority, including the present study, used lung cancer tissue and matching resected lung. ${ }^{6,9}$ Thus far, there is no consensus as to which species constitute the LCM and reminds one of the difficulties in validating gene expression signatures for diagnosis or prognosis.

Quality controls regarding the baseline level of bacterial diversity and abundance under sterile conditions are lacking, but guidelines are available. ${ }^{12}$ Remember the simian virus 40 studies and mesothelioma? ${ }^{13}$ Specimen handling could result in contamination with nonpathogenic bacteria at any point in this experiment. Are the patients intubated for the BALs? Was the scope at any time exposed to the supraglottic area? The same problems are magnified when trying to explore the local; that is, tissue microbiome, as opposed to the mucosal airway microbiome. How was the tissue handled? Did it go to pathology and then get distributed or was it harvested, cut, and placed in sterile vials at the operating table? Were the instruments at the table dipped in DNAase free water and subjected to the same next-generation sequencing and transcriptomic analyses to document the baseline level of contamination?

Reading the article impresses the reader with the complicated bioinformatics, which can make interpretation of the data difficult. Despite other investigators using these same techniques, a uniform approach for the bioinformatic analyses of microbiome data in the literature is missing. 
Modeling and interpretation of the data for these studies requires consensus amongst expert computational biologists and these techniques are ever-changing due to advances in artificial intelligence. ${ }^{14,15}$

The only way we are going to gain confidence in these studies is for surgeons (assuming the role of the sterility police) get involved with prospective collection of these specimens. Ideally, this is performed at the time of resection for lung cancer: intubate the patient, do the BAL DNAase free control through the bronchoscope, perform the BAL on the uninvolved side first (think about using the double lumen!), and finally take on the involved side. Harvest the upper airway microbiome with a Yankauer sucker while its isolated by the endotracheal tube from the lower airway. A small piece of tumor and matching lung should be immediately procured at the operating table, put it in a sterile vial, then snap-frozen in liquid nitrogen future sterile DNA/RNA extraction.

Finally, we need more mechanistic ${ }^{11}$ and in vivo studies ${ }^{16}$ that will help us validate that a specific bug or its toxins affect the local microenvironment to promote lung cancer. Once these preclinical models give insights into microbiome-influenced pathways to target, novel treatment or prevention strategies may be possible. Then maybe we can think about antibiotics.

\section{References}

1. Mao Q, Jiang F, Yin R, Wang J, Xia W, Dong G, et al. Interplay between the lung microbiome and lung cancer. Cancer Lett. 2018:415:40-8.

2. Patnaik SK, Cortes EG, Kannisto ED, Punnanitinont A, Dhillon SS, Liu S, et al. Lower airway bacterial microbiome may influence recurrence after resection of early-stage non-small cell lung cancer. J Thorac Cardiovasc Surg. 2021;161: 419-29.e16.

3. Wang L, Hao K, Yang T, Wang C. Role of the lung microbiome in the pathogenesis of chronic obstructive pulmonary disease. Chin Med J (Engl). 2017;130:2107-11.

4. Sulaiman I, Wu BG, Li Y, Scott AS, Malecha P, Scaglione B, et al. Evaluation of the airway microbiome in nontuberculous mycobacteria disease. Eur Respir J. 2018;52(4).

5. Naidoo CC, Nyawo GR, Wu BG, Walzl G, Warren RM, Segal LN, et al. The microbiome and tuberculosis: state of the art, potential applications, and defining the clinical research agenda. Lancet Respir Med. 2019;7:892-906.

6. Peters BA, Hayes RB, Goparaju C, Reid C, Pass HI, Ahn J. The microbiome in lung cancer tissue and recurrence-free survival. Cancer Epidemiol Biomarkers Prev. 2019:28:731-40.

7. Maddi A, Sabharwal A, Violante T, Manuballa S, Genco R, Patnaik S, et al. The microbiome and lung cancer. J Thorac Dis. 2019;11:280-91.

8. Kovaleva OV, Romashin D, Zborovskaya IB, Davydov MM, Shogenov MS, Gratchev A. Human lung microbiome on the way to cancer. I Immunol Res. 2019;2019:1394191.

9. Greathouse KL, White JR, Vargas AJ, Bliskovsky VV, Beck JA, von Muhlinen N, et al. Interaction between the microbiome and TP53 in human lung cancer. Genome Biol. 2018;19:123.

10. Gomes S, Cavadas B, Ferreira JC, Marques PI, Monteiro C, Sucena M, et al. Profiling of lung microbiota discloses differences in adenocarcinoma and squamous cell carcinoma. Sci Rep. 2019;9:12838.

11. Tsay JJ, Wu BG, Badri MH, Clemente JC, Shen N, Meyn P, et al. Airway microbiota is associated with upregulation of the PI3K pathway in lung cancer. Am J Respir Crit Care Med. 2018;198:1188-98.

12. Carney SM, Clemente JC, Cox MJ, Dickson RP, Huang YJ, Kitsios GD, et al Methods in lung microbiome research. Am J Respir Cell Mol Biol. 2020;62: 283-99.

13. Strickler HD, Goedert JJ, Devesa SS, Lahey J, Fraumeni JF Jr, Rosenberg PS Trends in U.S. pleural mesothelioma incidence rates following simian virus 40 contamination of early poliovirus vaccines. J Natl Cancer Inst. 2003;95:38-45.

14. Zhu Q, Li B, He T, Li G, Jiang X. Robust biomarker discovery for microbiomewide association studies. Methods. 2020;173:44-51.

15. Wang T, Yang C, Zhao H. Prediction analysis for microbiome sequencing data Biometrics. 2019;75:875-84.

16. Jin C, Lagoudas GK, Zhao C, Bullman S, Bhutkar A, Hu B, et al. Commensal microbiota promote lung cancer development via $\gamma \delta$ T cells. Cell. 2019;176: 998-1013.e16. 